\title{
Robust Estimation of Kinetic Parameters in Dynamic PET Imaging
}

\author{
Fei Gao ${ }^{1}$, Huafeng $\mathrm{Liu}^{2,1}$, and Pengcheng $\mathrm{Shi}^{1}$ \\ 1 Golisano College of Computing and Information Sciences, Rochester Institute of \\ Technology, Rochester, NY, 14623, USA \\ 2 State Key Laboratory of Modern Optical Instrumentation, Zhejiang University, \\ Hangzhou, 310027, China
}

\begin{abstract}
Dynamic PET imaging provides important information for biological research, clinical diagnosis and pharmacokinetic analysis through kinetic modeling and data-driven parameter estimation. Kinetic parameters quantitatively describe dynamic material exchange and metabolism of radiotracers in plasma and tissues. While many efforts have been devoted to estimate kinetic parameters from dynamic PET, the poor statistical properties of the measurement data in low count dynamic acquisition and the uncertainties in estimating the arterial input function have limited the accuracy and reliability of the kinetic parameter estimation. Additionally, the quantitative analysis of individual kinetic parameters is not yet implemented. In this paper, we present a robust kinetic parameter estimation framework which is robust to both the poor statistical properties of measurement data in dynamic PET and the uncertainties in estimated arterial input function, and is able to analyze every single kinetic parameter quantitatively. The strategy is optimized with robust $H_{\infty}$ estimation under minimax criterion. Experiments are conducted on Monte Carlo simulated data set for quantitative analysis and validation, and on real patient scans for assessment of clinical potential.
\end{abstract}

\section{Introduction}

Dynamic Positron Emission Tomography (PET) is a molecular imaging technique used to monitor the spatiotemporal distribution of a radiotracer in vivo and provides important information for biological research, clinical diagnosis and pharmacokinetic analysis 12]. PET offers good promise for accelerating the process from pre-clinical discover to Phase III studies of drug development, which benefits from various radiolabelled biochemicals and well established metabolism models. The accurate quantitative descriptions (kinetic parameters) of the interaction of the drug with a desired binding site and the neurotransmitter concentration will significantly advance the studies of drug discovery and development.

The typical approach of estimating kinetic parameters is first to reconstruct activity distributions, and then to fit the calculated time activity curve (TAC) to a predefined kinetic model. However, the practicability of this kind of approaches relies on the accuracy of image reconstruction. There are also many

G. Fichtinger, A. Martel, and T. Peters (Eds.): MICCAI 2011, Part I, LNCS 6891, pp. 492-499, 2011. (C) Springer-Verlag Berlin Heidelberg 2011 
efforts trying to estimate from projection data directly by introducing either linear or nonlinear models 3], however, the optimization algorithms are generally very complicated to implement. Kamasak et al. applied coordinate descent optimization but which is still limited in specific models 44. Wang et al. applied an generalized algorithm for reconstruction of parametric images [5]. However, in dynamic PET imaging, the low count data acquisition procedures make the statistical properties of measurement data very poor, moreover, the uncertainties introduced by various data corrections make the noise properties complicated, and will lead to a suboptimal estimation [6]. Additionally, difficulties in arterial sampling and uncertainties during input function fitting will result in errors in kinetic parameter estimation. Furthermore, the quantitative estimation and analysis of the dynamic change of individual kinetic parameter, which is not yet implemented, will definitely lead to better understandings of tissue biology [7.

In this paper, we concentrate on the development of a robust kinetic parameter estimation framework from projection data directly. PET measurement equation is successfully transformed and combined with kinetic models, which makes it very flexible to satisfy different kinetic models. The framework is robust to both poor statistical properties of measurement data and uncertainties in arterial input function estimation when no blood sampling exists, and is able to analyze every single kinetic parameter quantitatively. The strategy is optimized by robust $H_{\infty}$ filter under minimax criterion. Experiments are conducted with Monte Carlo simulated dynamic data for statistical analysis and validation, and on real patient scans for assessment of clinical potential.

\section{Method}

\subsection{Modeling of Tracer Kinetics}

In this paper, a general two-tissue three-compartment model is adopted to describe regional tracer kinetics as shown in Fig 1, where $C_{P}(\mathrm{pmol} / \mathrm{ml})$ is arterial concentration of radiotracer, $C_{F}$ and $C_{B}(\mathrm{pmol} / \mathrm{ml})$ are the concentrations of non-specific binding and specific binding tracers in tissues. Parameters $k_{1}, k_{2}, k_{3}$ and $k_{4}\left(\mathrm{~min}^{-1}\right)$ specify radiotracer transport rates. The time variation of kinetic model in voxel $i$ can be denoted by first-order differential equations as:

$$
\begin{aligned}
& \frac{d C_{F i}(t)}{d t}=k_{1 i}(t) C_{P i}(t)+k_{4 i}(t) C_{B i}(t)-\left(k_{2 i}(t)+k_{3 i}(t)\right) C_{F i}(t) \\
& \frac{d C_{B i}(t)}{d t}=k_{3 i}(t) C_{F i}(t)-k_{4 i}(t) C_{B i}(t)
\end{aligned}
$$

\subsection{Modeling of Dynamic PET Measurement with Tracer Kinetics}

Dynamic PET imaging involves a sequence of contiguous acquisition with different temporal resolutions, which can be formulated as a projection transform:

$$
y(t)=D x(t)+e(t)
$$




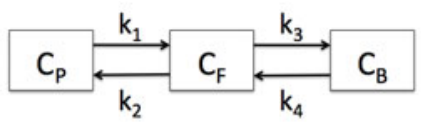

Fig. 1. Two-tissue three-compartment model

Here, $y(t)$ is the projection data and $x(t)=\left\{x_{i}(t) \mid i=1, \cdots, n\right\}^{T}$ is the activity concentration at time frame $t . n$ is the total number of voxels. $D$ is the system probability matrix. $e(t)$ is the overall measurement uncertainties. Here we will transform Eqn.(3) to accommodate kinetic models. Firstly, activity concentration $x$ will be the combination of $C_{F}$ and $C_{B}$, then Eqn.(3) will be

$$
y(t)=\left[\begin{array}{ll}
D & D
\end{array}\right]\left[\begin{array}{l}
C_{F}(t) \\
C_{B}(t)
\end{array}\right]+e(t)
$$

where $C_{F}(t)=\left\{C_{F i}(t) \mid i=1, \cdots, n\right\}^{T}$ and $C_{B}(t)=\left[C_{B i}(t) \mid i=1, \cdots, n\right\}^{T}$. After the dynamic change of measurement $\frac{d y_{i}(t)}{d t}$ being deduced, we substitute the differential equations Eqn.(11) and (2) and do a simple transformation to arrange 4 kinetic parameters $(k 1, k 2, k 3, k 4)$ in a column vector will yield

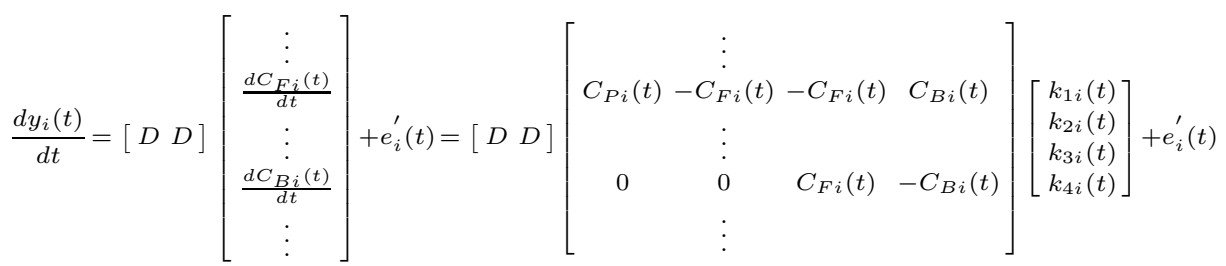

By denoting $R_{i}(t)=\left[\begin{array}{cccc}C_{P i}(t) & -C_{F i}(t) & -C_{F i}(t) & C_{B i}(t) \\ \vdots & & & \\ 0 & 0 & C_{F i}(t) & -C_{B i}(t) \\ \vdots & \end{array}\right]$ and $S_{i}(t)=\left[\begin{array}{c}k_{1 i}(t) \\ k_{2 i}(t) \\ k_{3 i}(t) \\ k_{4 i}(t)\end{array}\right]$,

we can get the dynamic change of total measurement data from all voxels as

$$
\begin{aligned}
\frac{d y(t)}{d t}=\sum_{i=1}^{n} \frac{d y_{i}(t)}{d t} & =\left[\begin{array}{ll}
D & D
\end{array}\right]\left[\begin{array}{lll}
R_{1}(t) \cdots & R_{i}(t) \cdots R_{n}(t)
\end{array}\right]\left[\begin{array}{c}
S_{1}(t) \\
\vdots \\
S_{i}(t) \\
\vdots \\
S_{n}(t)
\end{array}\right]+e^{\prime}(t) \\
& =\left[\begin{array}{ll}
D & D
\end{array}\right] R(t) S(t)+e^{\prime}(t)
\end{aligned}
$$

Now we have set up the relationship between the change of measurement data and kinetic parameters directly by Eqn.(6). 


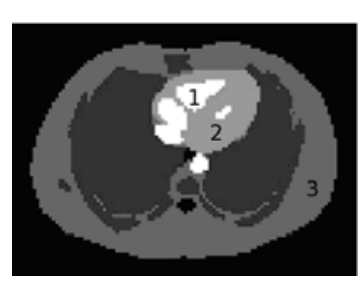

(a)

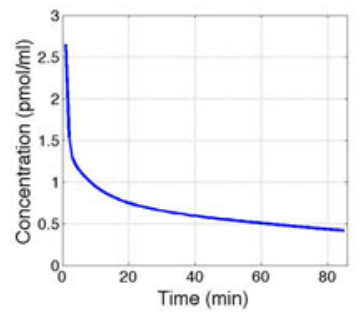

(b)

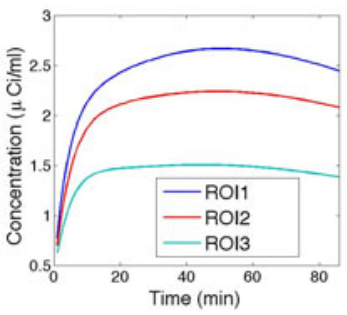

(c)

Fig. 2. (a) Zubal phantom; (b) Blood input function; (c) Output TACs of 3 ROIs

\subsection{Robust Solution by $\boldsymbol{H}_{\infty}$ Optimization Under Minimax Criterion}

Gao et al. 8 present an efficient and robust estimation framework for activity reconstruction of dynamic PET imaging. No statistical assumptions needed make it robust to the poor statistical properties in low count acquisition and system noises. We will transform our problem to fit that solution framework. Since kinetic parameters are generally assumed to be constant, we can set:

$$
S(t+1)=S(t)+v(t)
$$

Here, $v$ is possible disturbances. With Eqn.(6) and Eqn.(7), the corresponding minimax performance equation will be " $\min _{S \in L} \max _{e \in E, v \in V} F(S, e, v)$ ", where $L, E$ and $V$ are the sets of solutions, uncertainties of measurement and state transition. As an iterative solution, we also define a linear combination of $S(t)$ as " $z(t)=g(S(m), v(m))$ where $m=1,2 \ldots t "$, then objective function $J$ will be

$$
J=\frac{\sum\|z(t)-\hat{z}(t)\|_{Q(t)}^{2}}{\|S(0)-\hat{S}(0)\|_{p_{o}^{-1}}^{2}+\sum\left(\|v(t)\|_{V(t)^{-1}}^{2}+\|e(t)\|_{E(t)^{-1}}^{2}\right)}
$$

where the notation $\|x\|_{G}^{2}$ is defined as the square of the weighted (by $G$ ) $L_{2}$ norm of $x . p_{o}, E(t), V(t)$ and $Q(t)$ are weighting matrices. $\hat{S}(0)$ is the initialization of $x$. More detailed settings and initialization of parameters can be found in [9].

\section{Experiments}

\subsection{Monte Carlo Simulated Dynamic PET Data}

The first dynamic PET data set is from Monte Carlo simulation. The simulated PET scanner is Hamamatsu SHR74000. The phantom used here is Zubal thorax phantom. One sample slice is shown in Fig. 2(a). 3 regions including heart, muscle and chest wall are selected as ROI1-3. The experiment is a dynamic ${ }^{18} \mathrm{~F}-\mathrm{FDG}$ study with the compartment model in Sec 2.1 for imaging glucose metabolism. The TACs of 3 ROIs are generated by analytical Feng's input function:

$$
C_{P}^{F D G}(t)=\left(A_{1} t-A_{2}-A_{3}\right) e^{-\lambda_{1} t}+A_{2} e^{-\lambda_{2} t}+A_{3} e^{-\lambda_{3} t}
$$


Experiment 1

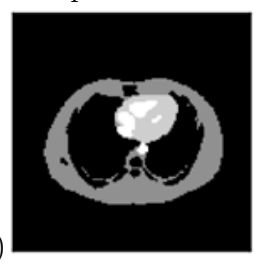

(a)

(b)
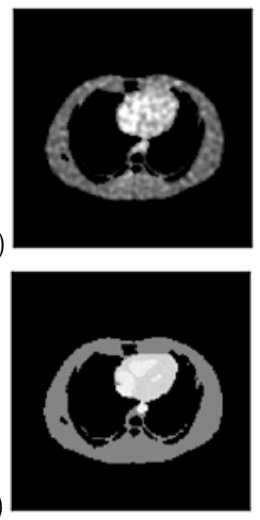

Experiment 2
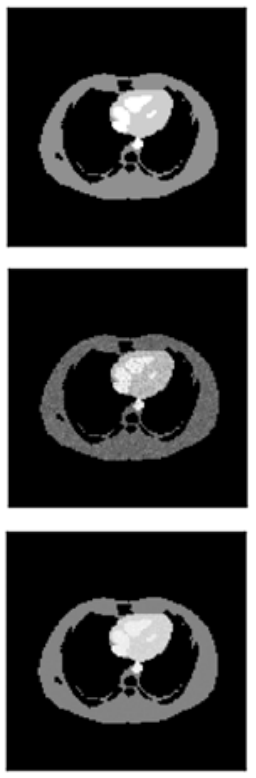

Experiment 3
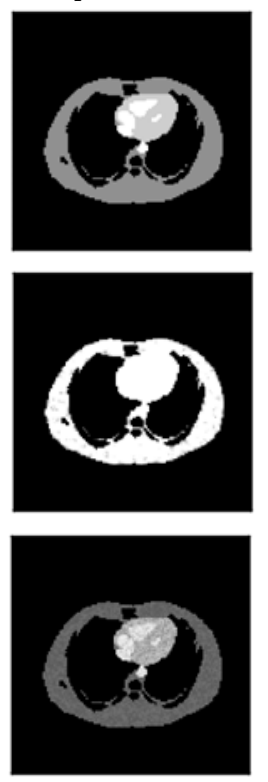

Experiment 4
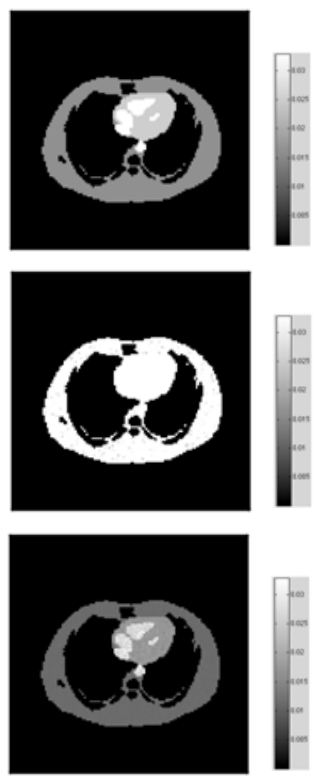

Fig. 3. Influx rate maps. (a) Ground Truth; (b) WLSCF; (c) Our Method.

The parameters $\lambda_{i}$ and $A_{i}$ used here are $A_{1}=85.112 \mu \mathrm{Ci} / \mathrm{mL} / \mathrm{min}, A_{2}=$ $2.081 \mu \mathrm{Ci} / \mathrm{mL}, A_{3}=2.188 \mu \mathrm{Ci} / \mathrm{mL}, \lambda_{1}=4.1339 \mathrm{~min}^{-1}, \lambda_{2}=0.01043 \mathrm{~min}^{-1}$ and $\lambda_{3}=0.1191 \mathrm{~min}^{-1}$. The dynamic acquisition consists of 85 frames: $15 \times 0.2 \mathrm{~min}$, $20 \times 0.5 \mathrm{~min}, 40 \times 1 \mathrm{~min}$ and $10 \times 3 \mathrm{~min}$. Kinetic parameters in simulations are, ROI1: 0.102, 0.130, 0.062, 0.0068; ROI2: 0.082, 0.102, 0.045, 0.004; ROI3: 0.064, 0124, 0.042, 0.0035. Calculated input function and TACs are shown in Fig 2 (b) and (c). The differences of total counts between time frames are around $1 \mathrm{k}$.

4 experiments (Experiment 1-4) are conducted under different conditions. Since input function is required and no blood sampling is desired, firstly, 4 experiments are divided into 2 groups: the first group (Experiment 1 and 2) uses a perfect input function, while the second group (Experiment 3 and 4) uses an imperfect input function generated by image-derived sampling method. Secondly, due to poor statistical properties of measurement data in low count dynamic PET imaging, spatial constrains are also introduced. In Experiment 1 and 3, every voxel is estimated separately. For a comparison, Experiment 2 and 4 are performed with "local average" constraints to improve statistical properties, which means the measurement data in one voxel is averaged by surrounding voxels. Besides our method, a typical Weighted Least Square Curve Fit (WLSCF) method provided by COMKAT toolbox, is conducted as comparison. To evaluate the experiments quantitatively, statistical analysis on estimated results against true values is performed. Let $N_{p}$ be the total number of voxels or ROIs, $S E_{i}$ and $S T_{i}$ be the estimation of $i$ th kinetic parameter and corresponding true value, then define Bias $=\frac{1}{N_{p}} \sum_{i=1}^{N_{p}} \frac{\left(S E_{i}-S T_{i}\right)}{S T_{i}}$ and $S t d=\sqrt{\frac{1}{N_{p}-1} \sum_{i=1}^{N_{p}}\left(\frac{S E_{i}-S T_{i}}{S T_{i}}\right)^{2}}$. We also 
Table 1. The calculated Bias and Std of Experiment 1 (top) and 2 (bottom)

\begin{tabular}{|c|c|c|c|c|c|}
\hline & Bias \pm Std & $\mathrm{k} 1$ & $\mathrm{k} 2$ & $\mathrm{k} 3$ & $\mathrm{k} 4$ \\
\hline ROI1 & WLSCF & $0.10706 \pm 0.21072$ & $0.17943 \pm 0.30803$ & $-0.09460 \pm 0.25205$ & $-0.26725 \pm 0.40846$ \\
\cline { 2 - 6 } & Our Method & $-0.04794 \pm 0.05450$ & $-0.16225 \pm 0.17368$ & $-0.20677 \pm 0.22111$ & $-0.14092 \pm 0.15652$ \\
\hline \multirow{2}{*}{ ROI2 } & WLSCF & $-0.01347 \pm 0.22397$ & $0.24409 \pm 0.32029$ & $-0.03134 \pm 0.22162$ & $0.16834 \pm 0.65988$ \\
\cline { 2 - 6 } & Our Method & $-0.00459 \pm 0.04190$ & $0.10929 \pm 0.11838$ & $0.18639 \pm 0.19926$ & $0.09558 \pm 0.14263$ \\
\hline ROI3 & WLSCF & $-0.16228 \pm 0.29534$ & $0.37212 \pm 0.47103$ & $0.07289 \pm 0.24139$ & $0.29611 \pm 0.81515$ \\
\cline { 2 - 6 } & Our Method & $-0.12107 \pm 0.14058$ & $-0.02826 \pm 0.08700$ & $0.00574 \pm 0.00614$ & $0.19748 \pm 0.21488$ \\
\hline
\end{tabular}

\begin{tabular}{|c|c|c|c|c|c|}
\hline & Bias \pm Std & $\mathrm{k} 1$ & $\mathrm{k} 2$ & $\mathrm{k} 3$ & $\mathrm{k} 4$ \\
\hline ROI1 & WLSCF & $0.00502 \pm 0.08444$ & $0.09966 \pm 0.12406$ & $-0.08475 \pm 0.11367$ & $-0.11420 \pm 0.20296$ \\
\cline { 2 - 6 } & Our Method & $-0.01904 \pm 0.02867$ & $-0.03211 \pm 0.03446$ & $-0.07005 \pm 0.07490$ & $0.10215 \pm 0.10967$ \\
\hline ROI2 & WLSCF & $-0.01809 \pm 0.08325$ & $0.21458 \pm 0.23228$ & $0.03688 \pm 0.09033$ & $0.13241 \pm 0.32270$ \\
\cline { 2 - 6 } & Our Method & $-0.06743 \pm 0.07298$ & $-0.03760 \pm 0.04064$ & $-0.00786 \pm 0.00840$ & $0.05390 \pm 0.05763$ \\
\hline ROI3 & WLSCF & $-0.16799 \pm 0.20050$ & $0.33093 \pm 0.34760$ & $0.05029 \pm 0.09786$ & $0.28117 \pm 0.42891$ \\
\cline { 2 - 6 } & Our Method & $-0.05638 \pm 0.06740$ & $0.08000 \pm 0.09407$ & $0.04364 \pm 0.04665$ & $-0.14543 \pm 0.15650$ \\
\hline
\end{tabular}

calculate the influx rate map (Rate) which is related to the glucose metabolic rate as $R$ ate $=\frac{k_{1} k_{3}}{k_{2}+k_{3}}$. Parametric maps also show visual comparisons.

Table 1 and 2 show calculated Bias and Std of 4 experiments in 2 groups. Fig 3 shows the calculated influx rate maps. Table 1 indicates that WLSCF can achieve acceptable results with perfect input function, and the local average constraint introduced in Experiment 2 improves Std of all estimated parameters by improving the statistical properties of measurement data. The Bias and Std of results from our methods are better than that from WLSCF. The results also show the robustness of our method in dealing with the poor statistical properties of measurement data. The statistical analysis in Table 2 shows that with input function from estimation, WLSCF leads to worse results indicated by increased Std values in both experiments, especially $k_{1}$ and $k_{4} \cdot k_{1}$ is the rate constant indicating the radiotracer exchange from arterial to tissue, which is highly affected by the accuracy of estimated input function, and $k_{4}$ is the FDG dephosphorylation rate and very sensitive to the system. The influx rate maps also show obvious overestimation of WLSCF. However, our method still achieves robust estimation results of all parameters in both experiments and yields acceptable Std with the imperfect input function. And the calculated Std shows that some parameter estimations by our method with imperfect input function are even comparable with that by WLSCF with perfect input function.

\subsection{Experiments with Data from Real Patient Scan}

The real patient data in this study is a dynamic PET scan acquired from a 28year-old, $75 \mathrm{~kg}$ male volunteer. The scanner used is Hamamatsu SHR-22000 whole body PET scanner. $10 \mathrm{mCi}{ }^{18} \mathrm{~F}-\mathrm{FDG}$ is injected and a dynamic acquisition of the thoracic cavity starts just after injection. The acquisition consists of 40 time frames: $20 \times 0.5 \mathrm{~min}, 15 \times 1 \mathrm{~min}$, and $5 \times 2 \mathrm{~min}$. The input function is estimated by the image-derived method. Since the influx rate maps have direct relationships 
Table 2. The calculated Bias and Std of Experiment 3 (top) and 4 (bottom)

\begin{tabular}{|c|c|c|c|c|c|}
\hline & Bias \pm Std & $\mathrm{k} 1$ & $\mathrm{k} 2$ & $\mathrm{k} 3$ & $\mathrm{k} 4$ \\
\hline \multirow{2}{*}{ ROI1 } & WLSCF & $1.05780 \pm 1.14375$ & $-0.09464 \pm 0.30377$ & $0.37156 \pm 0.68273$ & $0.12470 \pm 0.94807$ \\
\cline { 2 - 6 } & Our Method & $-0.27954 \pm 0.30441$ & $-0.15235 \pm 0.16289$ & $0.05914 \pm 0.06328$ & $-0.12877 \pm 0.13901$ \\
\hline \multirow{2}{*}{ ROI2 } & WLSCF & $1.25494 \pm 1.31110$ & $0.11049 \pm 0.38121$ & $0.45580 \pm 0.67132$ & $0.24023 \pm 1.13308$ \\
\cline { 2 - 6 } & Our Method & $-0.36468 \pm 0.39731$ & $-0.16074 \pm 0.17187$ & $0.21405 \pm 0.22883$ & $0.30306 \pm 0.32609$ \\
\hline \multirow{2}{*}{ ROI3 } & WLSCF & $1.20747 \pm 1.28191$ & $-0.12640 \pm 0.26492$ & $0.00213 \pm 0.28536$ & $0.48420 \pm 1.51799$ \\
\cline { 2 - 6 } & Our Method & $-0.39567 \pm 0.42691$ & $-0.13915 \pm 0.25402$ & $-0.05089 \pm 0.05440$ & $0.24891 \pm 0.27461$ \\
\hline
\end{tabular}

\begin{tabular}{|c|c|c|c|c|c|}
\hline & Bias \pm Std & $\mathrm{k} 1$ & $\mathrm{k} 2$ & $\mathrm{k} 3$ & $\mathrm{k} 4$ \\
\hline ROI1 & WLSCF & $1.14242 \pm 1.20911$ & $0.17117 \pm 0.33201$ & $0.66864 \pm 0.82730$ & $-0.17832 \pm 0.51223$ \\
\cline { 2 - 6 } & Our Method & $-0.19232 \pm 0.21434$ & $0.17155 \pm 0.18339$ & $0.22930 \pm 0.24531$ & $-0.06705 \pm 0.07176$ \\
\hline ROI2 & WLSCF & $1.24255 \pm 1.27450$ & $0.15610 \pm 0.28138$ & $0.50822 \pm 0.61257$ & $-0.00525 \pm 0.78607$ \\
\cline { 2 - 6 } & Our Method & $-0.26658 \pm 0.28651$ & $0.25757 \pm 0.27536$ & $0.12868 \pm 0.13816$ & $-0.26835 \pm 0.28689$ \\
\hline ROI3 & WLSCF & $1.25741 \pm 1.27605$ & $-0.13817 \pm 0.22765$ & $-0.00537 \pm 0.11263$ & $0.19770 \pm 1.04567$ \\
\cline { 2 - 6 } & Our Method & $-0.22002 \pm 0.23667$ & $-0.22338 \pm 0.24597$ & $-0.00636 \pm 0.00680$ & $0.16175 \pm 0.17297$ \\
\hline
\end{tabular}

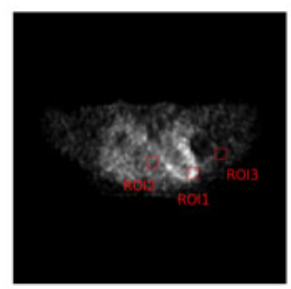

(a)

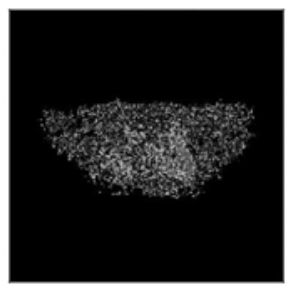

(b) WLSCF

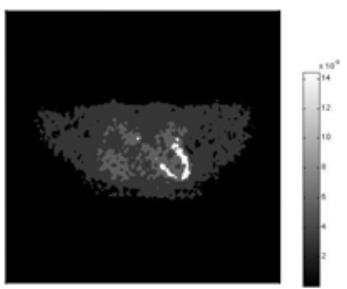

(c) Our Method

Fig. 4. (a) Reference activity map with 3 ROIs; (b), (c) Influx rate map

Table 3. Estimated kinetic parameters (top) and their MSE (bottom)

\begin{tabular}{|c|c|c|c|c|c|c|c|c|c|c|c|}
\hline \multicolumn{4}{|c|}{ ROI1 } & \multicolumn{4}{|c|}{ ROI2 } & \multicolumn{4}{|c|}{ ROI3 } \\
\hline $\mathrm{k} 1$ & $\mathrm{k} 2$ & k3 & $\mathrm{k} 4$ & $\mathrm{k} 1$ & $\mathrm{k} 2$ & k3 & $\mathrm{k} 4$ & $\mathrm{k} 1$ & $\mathrm{k} 2$ & $\overline{\mathrm{k} 3}$ & $\mathrm{k} 4$ \\
\hline 0.0805 & 0.4659 & 0.1020 & 0.0058 & 0.0493 & 0.9758 & 0.1198 & 0.0059 & 0.0094 & 0.1984 & 0.0973 & 0.0079 \\
\hline \multicolumn{4}{|c|}{ " } & \multicolumn{4}{|c|}{ " } & \multicolumn{4}{|c|}{ 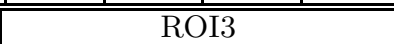 } \\
\hline $\mathrm{k} 1$ & $\mathrm{k} 2$ & k3 & $\mathrm{k} 4$ & $\mathrm{k} 1$ & $\mathrm{k} 2$ & k3 & $\mathrm{k} 4$ & $\mathrm{k} 1$ & $\mathrm{k} 2$ & k3 & $\mathrm{k} 4$ \\
\hline 0.1426 & 0.2030 & 0.1326 & 0.2018 & 0.2770 & 0.1438 & 0.1445 & 0.2047 & 0.3284 & 0.2236 & 0.1873 & 0.1989 \\
\hline
\end{tabular}

with activity distribution, so we choose an activity map from static scan as reference to extract ROIs. Fig. 4(a) shows the reference activity map and 3 ROIs are selected including left ventricular, heart muscle and body surface. Table 3 shows the estimated kinetic parameters by our method and their mean square error (MSE) in the 3 ROIs. The influx rate maps calculated by WLSCF and our method are shown in Fig 4 (b) and (c). Our method generates parametric maps with better contrast and MSEs are overall stable in 3 ROIs. 


\section{Conclusion}

A robust kinetic parameter estimation framework is presented, which is robust to both poor statistical properties in dynamic PET and uncertainties in arterial input function, and is able to analyze every single kinetic parameter.

Acknowledgement. This work is supported in part by the National Basic Research Program of China (No: 2010CB732504) and by the Department of Science and Technology of Zhejiang Province(No: 2010C33026).

\section{References}

1. Bailey, D., Townsend, D., Valk, P., Maisey, M.: Positron Emission Tomography: Basic Sciences. Springer, Heidelberg (2005)

2. Visser, E., Philippens, M., Kienhorst, L., Kaanders, J.: Comparison of tumor volumes derived from glucose metabolic rate maps and SUV maps in dynamic $18 \mathrm{~F}$ FDG PET. Journal of Nuclear Medicine 49(6), 892 (2008)

3. Tsoumpas, C., Turkheimer, F., Thielemans, K.: A survey of approaches for direct parametric image reconstruction in emission tomography. Medical Physics 35, 3963 (2008)

4. Kamasak, M.E., Bouman, C.A., Morris, E.D., Sauer, K.: Direct reconstruction of kinetic parameter images from dynamic pet data. IEEE Transactions on Medical Imaging 25, 636-650 (2005)

5. Wang, G., Qi, J.: Generalized algorithms for direct reconstruction of parametric images from dynamic PET data. IEEE Transactions on Medical Imaging 28(11), 1717-1726 (2009)

6. Gunn, R.: Tracer Kinetic Modeling via Basis Pursuit. In: Senda, M., Kimura, Y., Herscovitch, P. (eds.) Brain Imaging using PET. Academic Press, New York (2002)

7. Cobelli, C., Foster, D., Toffolo, G.: Tracer Kinetics in Biomedical Research: From Data to Model. Kluwer Academic/Plenum Publishers, New York (2000)

8. Gao, F., Liu, H., Shi, P.: Efficient Robust Reconstruction of Dynamic PET Activity Maps with Radioisotope Decay Constraints. In: Jiang, T., Navab, N., Pluim, J.P.W., Viergever, M.A. (eds.) MICCAI 2010. LNCS, vol. 6363, pp. 571-578. Springer, Heidelberg (2010)

9. Simon, D.: Optimal state estimation: Kalman, $\mathrm{H}_{\infty}$ and nonlinear approaches. John Wiley and Sons, Chichester (2006) 